\title{
ILUSTRAÇÃO DE CARACTERES MICROSCÓPICOS DE DROGAS VEGETAIS PARA O CONTROLE DE QUALIDADE FARMACOGNÓSTICO II. ECHINODORUS MACROPHYLLUS E RUTA GRAVEOLENS
}

\section{Illustration of vegetal drug MICROSCOPIC CHARACTERS FOR THE PHARMACOGNOSTIC QUALITY CONTROL II. Echinodorus macrophyllus E Ruta graveolens}

DUARTE, M. do R.

RECEBIDO: JUN/07 ACEITE: JUL/07

Laboratório de Farmacognosia, Departamento de Farmácia, Universidade Federal do Paraná (UFPR), Av. Pref. Lothário Meissner, 632, Jardim Botânico, 80210-170, Curitiba, PR, marciard@ufpr.br

\begin{abstract}
RESUMO
A etapa inicial do controle de qualidade de drogas vegetais farmacopéicas consiste na confirmação da identidade das mesmas, com base em descrições macro e microscópicas constantes em monografias. Quando essas descrições são complementadas com ilustrações do material em análise, o reconhecimento das estruturas vegetais é facilitado. Desse modo, este trabalho objetivou fornecer um recurso adicional para a caracterização de duas drogas farmacopéicas: chapéu-de-couro [Echinodorus macrophyllus (Kunth) Micheli, Alismataceae] e arruda (Ruta graveolens L., Rutaceae). O material botânico foi fixado, emblocado em glicol metacrilato, seccionado em micrótomo e corado. Dentre os caracteres anatômicos ilustrados, destacam-se em E. macrophyllus folha anfiestomática, mesofilo homogêneo e dutos secretores. Em R. graveolens, são evidentes folha glabra, mesofilo dorsiventral e cavidades secretoras. Os caracteres indicados favorecem a identificação dessas drogas vegetais.
\end{abstract}

Palavras-chave: Droga vegetal; farmacopéia; ilustração; microscopia.

\begin{abstract}
The first essay of the quality control of vegetal drugs from pharmacopoeias consists of their identification, based on macro and microscopic descriptions in monographs. When those descriptions are accompanied by illustrations of the material, the vegetal structures are easily recognized. Therefore, this work has aimed to supply an additional resource for the characterization of two vegetal drugs from pharmacopoeias: Echinodorus macrophyllus (Kunth) Micheli, Alismataceae and Ruta graveolens L., Rutaceae. The botanical material was fixed, embedded in glycol methacrylate, sectioned by microtome and stained. Among the illustrated anatomical characters, in E. macrophyllus it is emphasized amphistomatic leaf, homogeneous mesophyll and secretory ducts. In R. graveolens glabrous leaf, dorsiventral mesophyll and secretory cavities are evident. The indicated characters favour the identification of these vegetal drugs.
\end{abstract}

Key words: illustration; microscopy; pharmacopoeia; vegetal drug.

\section{INTRODUÇÃO}

O controle de qualidade de drogas vegetais farmacopéicas consiste na confirmação da identidade das mesmas, com base em descrições macro e microscópicas, seguindo-se então a pesquisa qualitativa e quantitativa de marcadores químicos e/ou princípios ativos, a determinação de impurezas e, quando aplicável, a pesquisa de falsificação. 
A descrição dos caracteres morfológicos externos (macroscópicos) e anatômicos (microscópicos) é elaborada utilizando-se nomenclatura botânica própria, com a qual alguns profissionais que executam o controle de qualidade têm pouca familiaridade. Quando essa descrição é complementada com ilustrações do material em análise, o reconhecimento das estruturas vegetais é facilitado sobremaneira. Levando-se em conta essa constatação, as publicações mais recentes da F. Bras. IV (FARMACOPÉIA, 1988-2003) apresentam figuras correspondentes às descrições morfológicas de algumas drogas vegetais.

Entretanto, diversas monografias não incluem ilustrações das estruturas vegetais. Desse modo, objetivando fornecer um recurso adicional para a caracterização de amostras a serem analisadas no controle de qualidade farmacognóstico, este trabalho registrou por meio de fotografias os aspectos anatômicos de duas drogas farmacopéicas: chapéu-de-couro e arruda. Esta é definida como sendo constituída pela planta florida de Ruta graveolens L., Rutaceae (SILVA, 1926). Apresenta odor marcante, porte subarbustivo, com caule ereto, folhas compostas verde-azuladas e inflorescências terminais amarelas (LORENZI; MATOS, 2002). Na medicina popular, a arruda é usada como anti-helmíntico, antiinflamatório, analgésico e antiespasmódico, sendo considerada abortivo em doses elevadas (PACIORNIK, 1990; DI-STASI; HIRUMA-LIMA, 2002; SKIDMORE-ROTH, 2004). Dentre os grupos de compostos químicos investigados, encontram-se alcalóides, flavonóides, cumarinas e óleo essencial (SKIDMORE-ROTH, 2004).

O chapéu-de-couro consta da F. Bras. I (SILVA, 1926) e F. Bras. II (FARMACOPÉIA, 1959) e consiste de folha de Echinodorus macrophyllus (Kunth) Micheli, Alismataceae. É uma planta aquática, perene, também conhecida vulgarmente como chá-mineiro, erva-do-pântano e erva-do-brejo, e da qual já foram isolados alcalóides, diterpenóides, esteróides e flavonóides (LORENZI; MATOS, 2002; AMARAL et al., 2005). Na terapêutica caseira, é empregada como diurético e anti-reumático (PACIORNIK, 1990; LORENZI; MATOS, 2002).

\section{MATERIAL E MÉTODOS}

Amostras de folhas adultas de E. macrophyllus e R. graveolens foram coletadas de exemplares identificados no Departamento de Botânica, do Setor de Ciências Biológicas, da Universidade Federal do Paraná, em 2002.

Fragmentos do material foliar foram fixados em FAA 70 (JOHANSEN, 1940), o qual foi substituído por etanol a 70 \% (BERLYN; MIKSCHE, 1976). Procedeu-se à confecção de laminário permanente, realizando-se inclusão dos fragmentos em glicol metacrilato (FEDER; O'BRIEN, 1968), seccionamento em micrótomo rotatório e coloração com azul de astra e fucsina básica (BRITO; ALQUINI, 1996).

O registro das estruturas foi feito por meio de fotomicrografias em papel obtidas com câmera acoplada a microscópio fotônico. As fotos coloridas, com as respectivas escalas micrométricas, foram analisadas, digitalizadas e transformadas em tons de cinza.

Nas ilustrações, a indicação dos caracteres anatômicos foliares foi feita com base nas descrições microscópicas adaptadas das monografias de chapéu-de-couro da F. Bras. I (SILVA, 1926) e F. Bras. II (FARMACOPÉIA, 1959), e arruda da F. Bras. I (SILVA, 1926).

\section{RESULTADOS E DISCUSSÃO}

\section{Chapéu-de-couro}

Em E. macrophyllus, a epiderme foliar não apresenta pêlos (tricomas) tectores ou glandulares, sendo classificada como glabra. Em vista frontal, as células epidérmicas mostram-se com contorno levemente sinuoso e paredes finas na região internervural, e assumem formato retangular, alongado e em disposição paralela na direção das nervuras. Numerosos estômatos circundados por duas a quatro células não diferenciadas estão presentes em ambas as faces epidérmicas (Fig. 1C, 1D), caracterizando a folha como anfiestomática.

O mesofilo tende a homogêneo (Fig. 1A, 1B, 1F), sendo constituído de um parênquima frouxo, de células ovais ou arredondadas, de paredes irregulares (parênquima plicado) (Fig. 1B, 1C). A nervura mediana é biconvexa, de forma um tanto quadrangular na superfície abaxial. Apresenta vários feixes vasculares de contorno circular, envoltos por um parênquima fundamental, formado de células isodiamétricas, dispostas de modo a deixar entre elas grandes lacunas. O mesofilo e o tecido fundamental da nervura são desprovidos de cristais.

Esses caracteres anatômicos, descritos na F. Bras. I (SILVA, 1926) e F. Bras. II (FARMACOPÉIA, 1959), são ilustrados neste trabalho, havendo ainda a indicação da presença de dutos secretores (Fig. 1A, 1E-1G) na 
folha de E. macrophyllus, os quais não são mencionados nas monografias farmacopéicas. Todavia, essas estruturas de secreção interna são descritas para o pecíolo dessa mesma espécie por LEITE et al. (2007) e para outros representantes de Echinodorus por MATIAS et al. (2007). Comparativamente aos resultados apresentados por LEITE et al. (2007) para o chapéu-de-couro, existem discordâncias anatômicas, particularmente pelo fato de descreverem folha hipoestomática, tricomas tectores e mesofilo dorsiventral.

Na opinião de VOGELMANN et al. (1996) e JOHNSON et al. (2005), a diferenciação de estruturas foliares sofre em grande parte influência do ambiente. Desse modo, espécies submetidas à intensa radiação luminosa tendem à simetria fotossintética, ou seja, desenvolvem parênquima paliçádico em ambas as faces epidérmicas e são anfiestomáticas. Por outro lado, aquelas sujeitas à menor luminosidade, geralmente apresentam mesofilo dorsiventral e estômatos exclusivamente na face abaxial da epiderme foliar. Reforçando o papel do ambiente na diferenciação estrutural, MATIAS et al. (2007) salientam que, pelo fato de as espécies de Echinodorus serem plantas aquáticas anfíbias, exibem plasticidade fenotípica para se adaptarem a terrenos alagadiços temporários em áreas semi-áridas. Como conseqüência, aqueles exemplares submersos tendem a apresentar mesofilo homogêneo e os emersos, dorsiventral (AMARAL et al., 2005).

\section{Arruda}

A folha de $R$. graveolens é glabra e as células epidérmicas de ambas as faces, em vista frontal, são sinuosas. Os estômatos são ladeados por três ou quatro células irregulares, sendo raros na superfície adaxial e abundantes na face oposta (Fig. 2A, 2D).

O mesofilo é heterogêneo assimétrico (dorsiventral) (Fig. 2A, 2B), constituído por duas camadas de células em paliçada na parte superior e por duas ou três fileiras de células racemosas (parênquima esponjoso) na inferior. As duas zonas encerram nódulos (cavidades) secretores esquizolisígenos (Fig. 2A, 2C) e o parênquima lacunoso (esponjoso) pode conter cristais estelares (drusas) de oxalato de cálcio.

A nervura mediana é biconvexa; o feixe vascular possui formato elíptico e ocorre aposto ao floema um periciclo celulósico, cujas células são poligonais e de paredes pouco espessas. O feixe vascular é envolvido por um tecido fundamental, que também contém nódulos (cavidades) secretores.

Os caracteres anatômicos foliares de R. graveolens são compatíveis com a família Rutaceae (METCALFE; CHALK, 1950), especialmente quanto à ocorrência de mesofilo dorsiventral e cavidades secretoras. Estas, segundo esses mesmos autores, foram interpretadas ora como esquizógenas, ora como lisígenas na família e estão sempre presentes no mesofilo.

Embora não tenha sido descrita na F. Bras. I (SILVA, 1926), a face abaxial da epiderme de R. graveolens é formada por células papilosas, como ilustrado neste trabalho (Fig. 2A, 2B). Essas células que assumem tal formato peculiar, em razão da convexidade das paredes periclinais externas, são consideradas comuns em Rutaceae (METCALFE; CHALK, 1950). Segundo VOGELMANN et al. (1996), as células papilosas intensificam a captação da luz difusa que chega à lâmina foliar e amplificam a transmissão da mesma ao clorênquima, favorecendo a fotossíntese.

Em conclusão aos aspectos gerais apresentados, a ilustração anatômica das espécies investigadas facilita a identificação da droga vegetal, ao se constituir em um recurso didático e de fácil consulta aos profissionais que realizam análises de controle de qualidade. 


\section{REFERÊNCIAS}

AMARAL, A. C. F.; SIMÕES, E. V.; FERREIRA, J. L. P. Coletânea científica de plantas de uso medicinal. Rio de Janeiro: Fiocruz, 2005.

BERLYN, G. P.; MIKSCHE, J. P. Botanical microtechnique and cytochemistry. Ames: lowa State University Press, 1976. BRITO, J. F. A.; ALQUINI, Y. A new method for staining botanical material embedded in glycol methacrylate (GMA). Braz. Arch. Biol. Technol., Curitiba, v. 39, p. 949-51, 1996.

DI-STASI, L. C.; HIRUMA-LIMA, C. A. Plantas medicinais na amazônica e na mata atlântica. São Paulo: Unesp, 2002. FARMACOPÉIA Brasileira. 4 ed. São Paulo: Atheneu, 1988-2003.

FARMACOPÉIA dos Estados Unidos do Brasil. 2 ed. São Paulo: Siqueira, 1959.

FEDER, N.; O’BRIEN, T. P. Plant microtechnique: some principles and new methods. Am. J. Bot., St. Louis, v. 55, p. 123-42, 1968.

JOHANSEN, D. A. Plant microtechnique. New York: McGraw-Hill Book, 1940.

JOHNSON, D. M.; SMITH, W. K.; VOGELMANN, T. C.; BRODERSEN, C. R. Leaf architecture and direction of incident light influence mesophyll fluorescence profiles. Am. J. Bot., St. Louis, v. 92, p. 1425-31, 2005.

LEITE, J. P. V.; PIMENTA, D. S.; GOMES, R. S. D. L.; DANTAS-BARROS, A. M. Contribuição ao estudo farmacobotânico da Echinodorus macrophyllus (Kunth) Micheli (chapéu-de-couro) - Alismataceae. Braz. J. Pharmacogn., João Pessoa, v. 17, p. 242-8, 2007.

LORENZI, H.; MATOS, F. J. A. Plantas medicinais do Brasil: nativas e exóticas. Nova Odessa: Plantarum, 2002.

MATIAS, L. Q.; SOARES, A.; SCATENA, V. L. Systematic consideration of petiole anatomy of species of Echinodorus Richard (Alismataceae) from North-eastern Brazil. Flora, Jena, v. 202, p. 395-402, 2007.

METCALFE, C. R.; CHALK, L. Anatomy of the dicotyledons: leaves, stem, and wood in relation to taxonomy, with notes on economic uses. Oxford: Clarendon Press, 1950.

PACIORNIK, E. F. A planta nossa de cada dia. Curitiba: Copygraf, 1990.

SILVA, R. A. D. Pharmacopéia dos Estados Unidos do Brasil. São Paulo: Nacional, 1926.

SKIDMORE-ROTH, L. Handbook of herbs and natural supplements. 2nd. ed. St. Louis: Mosby, 2004.

VOGELMANN, T. C.; NISHIO, J. N.; SMITH, W. K. Leaves and light capture: light propagation and gradients of carbon fixation within leaves. Trends Plant Sci., Oxford, v. 1, p. 65-70, 1996. 


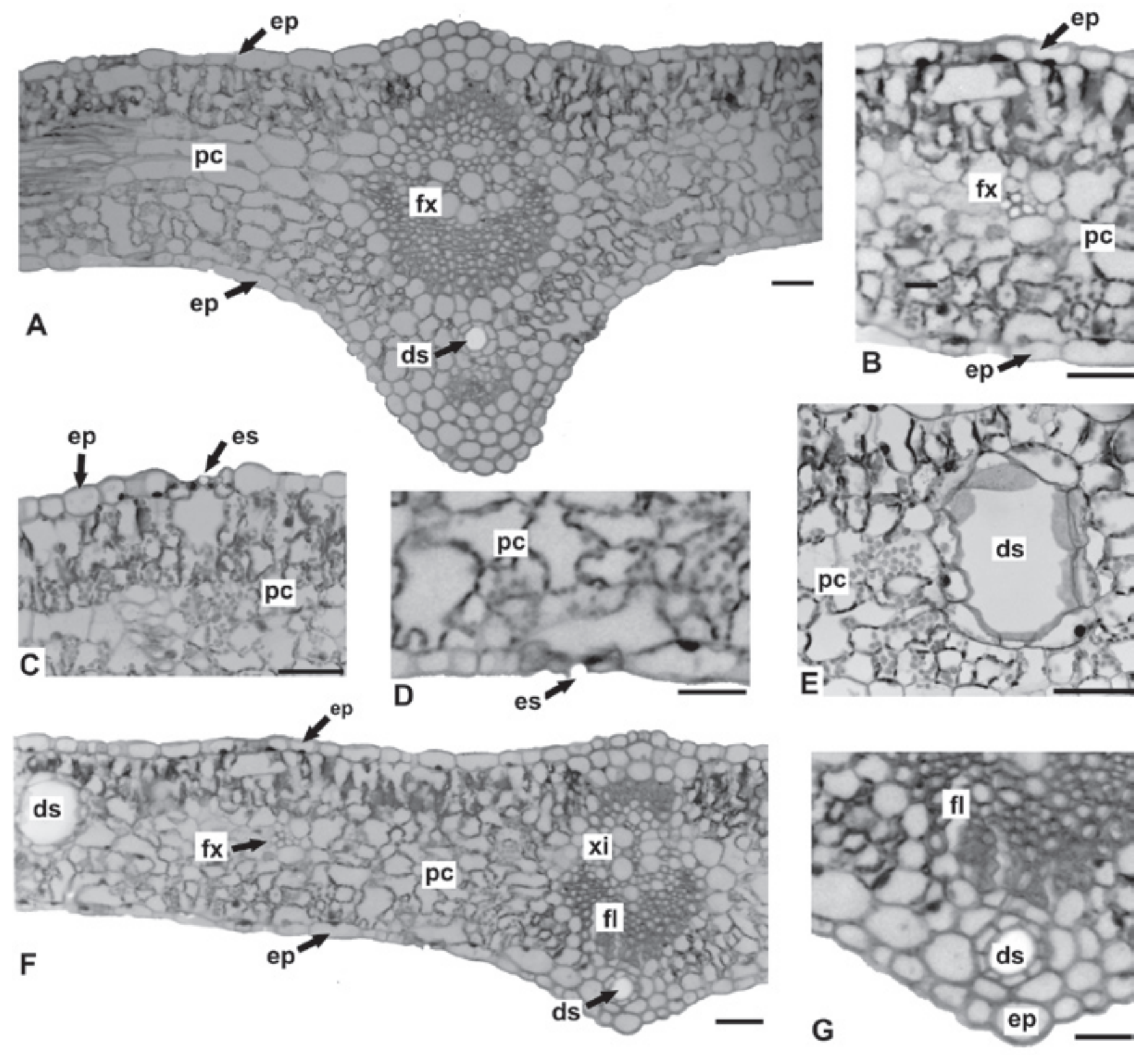

FIGURAS 1A-G. CHAPÉU-DE-COURO [ECHINODORUS MACROPHYLLUS (KUNTH) MICHELI, ALISMATACEAE] - SECÇÃO TRANSVERSAL DA FOLHA: A. NERVURA LATERAL E PARTE DA REGIÃO INTERNERVURAL; B. PORMENOR DO MESOFILO E DE UM FEIXE VASCULAR DE PEQUENO PORTE; C, D. DETALHE DA INSERÇÃO DE ESTÔMATOS NAS FACES ADAXIAL E ABAXIAL, RESPECTIVAMENTE; E. PORMENOR DE UM DUTO SECRETOR NO MESOFILO; F. REGIÃO INTERNERVURAL E NERVURA LATERAL, EVIDENCIANDO DUTOS SECRETORES; G. DETALHE DA FIGURA ANTERIOR. DS - DUTO SECRETOR, EP - EPIDERME, ES - ESTÔMATO, FL - FLOEMA, FX - FEIXE VASCULAR, PC - PARÊNQUIMA CLOROFILIANO, XI - XILEMA. $\mathrm{BARRA}=50 \mu \mathrm{m}$ 

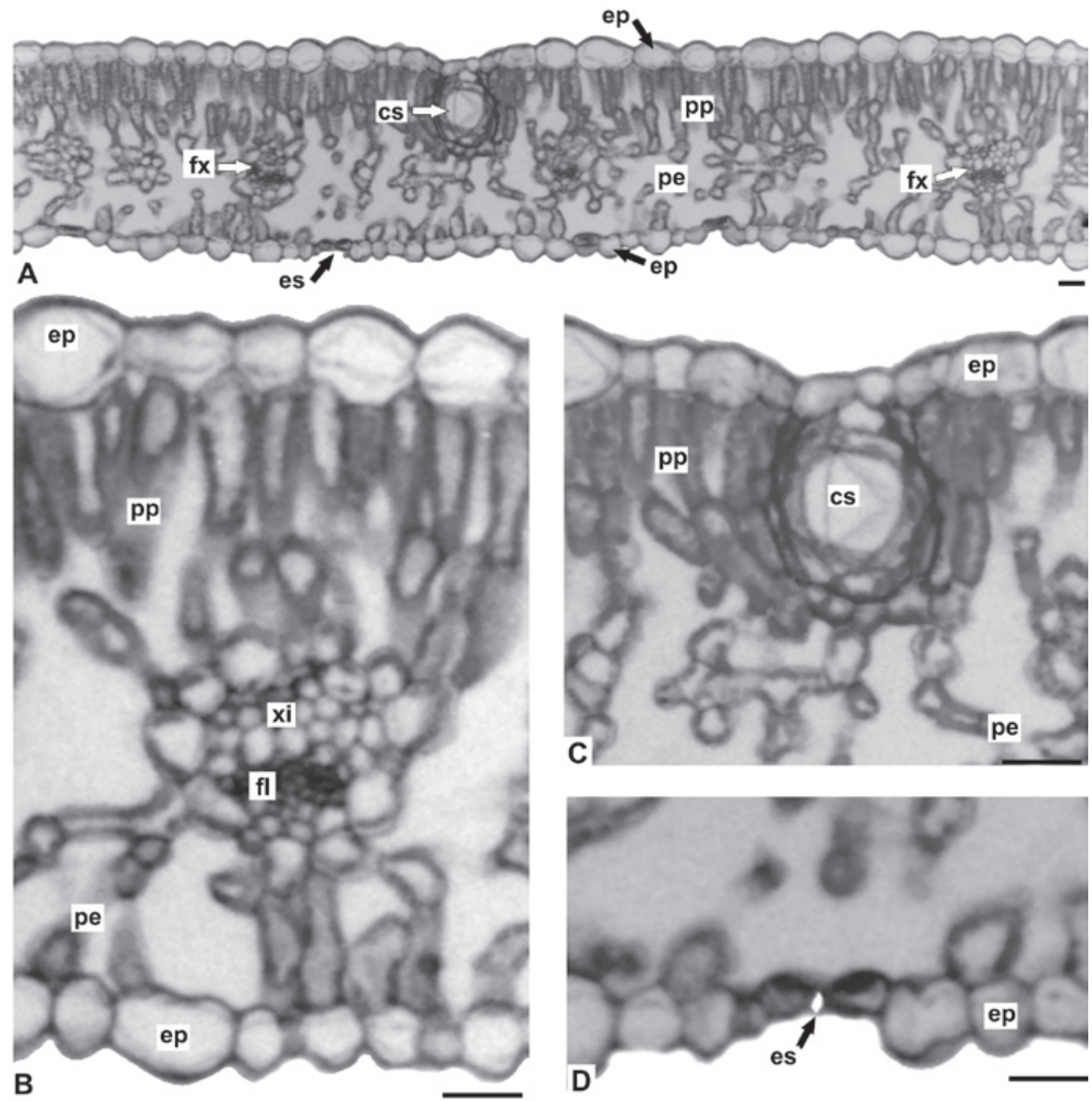

FIGURAS 2A-D. arruda (RUTA GRAVEOLENS L., RUTACEAE) - SECÇÃO TRANSVERSAL DA FOLHA: A. REGIÃO INTERNERVURAL, EM QUE SE NOTA O MESOFILO DORSIVENTRAL; B. PORMENOR DA FIGURA ANTERIOR, DESTACANDO UM FEIXE VASCULAR DE PEQUENO PORTE; C. DETALHE DE UMA CAVIDADE SECRETORA NO MESOFILO; D. ESTÔMATO LOCALIZADO NA FACE ABAXIAL. CS - CAVIDADE SECRETORA, ep - epiderme, es - estÔmato, fl - floema, $f x$ - feixe vascular, $p E$ - parênquima ESPONJOSO, PP - PARÊNQUIMA PALIÇÁDICO, xi - xilema. BARRA $=20 \mu \mathrm{m}$ 